\title{
Quantum measurement and geometry
}

\author{
James T. Wheeler* \\ Department of Physics and Astronomy, Swarthmore College, Swarthmore, Pennsylvania 19081 \\ (Received 26 May 1989)
}

\begin{abstract}
A model for the interpretation of spacetime as a Weyl geometry is proposed, based on the hypothesis that a system moves on any given path with a probability which is inversely proportional to the resulting change in length of the system. The results of physical measurements are calculated as the product of Weyl-conjugate gauge-dependent probabilities for the detection of conjugate objects. Each probability, expressed as a Wiener integral, is the Green's function for a diffusion equation. If the line integral of the Weyl field equals the action functional divided by $\hbar$, this equation provides the stochastic equivalent of the Schrödinger equation, with expanding and contracting states replacing the oscillating states of standard quantum theory. These dilatations are not directly measurable within the theory, but the rates of expansion and contraction are. We establish the classical limit of the theory by proving that the most general form of the Weyl field which gives an extremum of the action is restricted to be of a special form. Particles obeying the equations of motion for these extremal Weyl fields experience no measurable dilatation. However, the Weyl field itself is classically detectable, and gives a correct description of the electromagnetic interaction of a point particle.
\end{abstract}

\section{INTRODUCTION}

Almost immediately after the appearance of general relativity, Weyl ${ }^{1}$ proposed a generalization of Riemannian geometry which he felt could contain electromagnetism in a nontrivial way as the gauge field of local scale changes. In a conformally invariant geometry, this gauge field is a vector $W_{\mu}$ which Weyl equated to a multiple of the electromagnetic vector potential. Weyl showed that a conformal transformation changes $W_{\mu}$ by the gradient of a scalar and that such a transformation of the electromagnetic potential leaves the physical fields unaltered. With this interpretation, an electromagnetic field produces local scale changes in the same way that matter in general relativity causes curvature. Parallel transport of a vector in a Riemannian geometry rotates the vector, while in a Weyl geometry a vector transported around a closed path returns stretched as well as rotated.

Within a decade, two important facts were recognized concerning Weyl's theory. First, Einstein ${ }^{2}$ pointed out that the theory required atoms passing through different electromagnetic fields to have different sizes and hence different characteristic frequencies. This conflicts with experiments showing well-defined frequencies of emission for various atoms. The spreading of spectral lines is many orders of magnitude below what would be produced by the size changes predicted by the Weyl theory.

Second, and somewhat later, London ${ }^{3}$ showed that the ratio of the Weyl scale factor to the Schrödinger wave function is constant if the proportionality constant between the Weyl potential and the electromagnetic potential is taken to be imaginary. This observation gave birth to modern gauge theories. The original Weyl theory was absorbed into quantum mechanics with the original scale freedom becoming invariance under unitary gauge trans- formations.

The London paper marks a turning point in twentieth century physics. In retrospect, both the Weyl theory and the Schrödinger theory describe the evolution of a field in time and, given the factor of $i$ and the Kaluza-Klein framework used by London, those evolutions are the same. In the Weyl picture the field characterizes the length scales of fundamental matter, while in the Schrödinger picture it is the wave function corresponding to a fundamental particle.

In this paper we pursue this analogy further, noting a number of parallels between the description of measurement provided by wave mechanics and the description of measurement in a Weyl geometry. Based on this analogy we suggest a new interpretation of the Weyl vector which is consistent with atomic and macroscopic measurement, which retains the geometric characterization of electromagnetism inherent in Weyl's original theory and which provides new insight into the quantum description of nature.

Before describing the analogies of measurement, we stress particularly the equivalence between Weyl measurement and quantum measurement. A complete theory of measurement in a Weyl geometry contains the crucial elements of quantization, so that independent introduction of operators and commutation relations into this geometry is unnecessary and incorrect. We will show that quantization and uncertainty of measurement arise in a natural way from simple assumptions about the nature of motion in a Weyl geometry.

The close relationship between the time evolution of a quantum state according to the Schrödinger equation and the time evolution of a fundamental length in a Weyl geometry was noted above. But this is only the beginning of the correspondence between the two pictures. We provide here a partial comparison: 


\section{Property}

\section{Observable}

Equation of motion

Invariant formulation

Primary quantity

Dual quantity

Physical distribution

Principal invariance
Weyl-quantum correspondences Weyl geometry

Zero-Weyl-weight real number

Diffusion equation

Wiener path integral

Weightful length field, $\psi_{W}$

Weyl conjugate, $\psi_{-W}$

Probability $\psi_{W} \psi_{-W}$

$\psi_{W} \rightarrow e^{\omega \phi} \psi_{W}$ (conformal)
Quantum mechanics

Real eigenvalue

Schrödinger equation

Feynman path integral

Complex state function, $\psi$

Complex conjugate, $\psi^{*}$

Probability $|\psi|^{2}$

$\psi \rightarrow e^{i \phi} \psi$ (unitary)
Significantly, the superposition principle will hold in both pictures because of the linearity of the field equations, and Bell inequalities ${ }^{4}$ will be violated in both pictures because the physical probability is computed as the conjugate square of the time-evolved field.

The ultimate goal of this transcription, beyond the insight to be gained from a fresh look at the "meaning" of quantum-mechanical predictions, is to give an interpretation of those predictions which has direct geometrical sense and thereby provide a basis for a truly unified theory of the fundamental forces.

Section II begins with a brief review of some of the main elements of Weyl geometry, focusing principally on the Weyl vector, Weyl weight, and conjugacy. While these topics are all we shall require for our discussion more complete references are readily available. In Sec. III we state the basic assumptions we make concerning measurement in Weyl geometry. Then a brief study of the nonrelativistic quantum limit in Sec. IV leads to a physical interpretation of the Weyl vector. Section V makes use of this interpretation to show how the classical limit of the theory emerges when the Weyl field is restricted to a form that admits the possibility of preferred paths. It is further shown that this same restricted form of the Weyl field follows from an axiomatic approach to the world geometry. The final section provides a concise summary.

\section{WEYL GEOMETRY}

Our outline of Weyl geometry is based on Refs. 5 and 6 , where further detail is available. We begin with a conformal manifold, $(\mathcal{M},[g])$, where $\mathcal{M}$ is a real, fourdimensional manifold and $[g]$ is a conformal equivalence class of Lorentz metrics. We desire a Weyl spacetime rather than a Riemannian spacetime, which means that in addition to the usual local coordinate transformations we will have Weyl (conformal) transformations. On an arbitrary tensor field $T(x)$, the effect of the Weyl transformation is given by

$$
T(x)^{\prime}=e^{w(T) \Lambda(x)} T(x),
$$

where the Weyl weight $w(T)$ is a real number characterizing the Weyl action on $T$. We assume a coordinate basis $E_{\alpha}=\partial / \partial x^{\alpha}, E^{\alpha}=d x^{\alpha}$ in the tangent space and the dual space of one-forms to satisfy

$$
w\left(E_{\alpha}\right)=w\left(E^{\alpha}\right)=0 .
$$

In addition we define a torsion-free derivation $D$, which is

(1) linear: $D\left(a T_{1}+b T_{2}\right)=a D T_{1}+b D T_{2}$ for real numbers $a$ and $b$;

(2) Leibnitz: $D\left(T_{1} T_{2}\right)=\left(D T_{1}\right) T_{2}+T_{1}\left(D T_{2}\right)$;

(3) Weyl covariant: $D(f T)=[d f+w(f) W f] T+f D T$ where $W$ is a real one-form called the Weyl potential;

(4) zero weight: $w(D T)=w(T)$.

Under a Weyl transformation $W$ transforms as $W^{\prime}=W-d \Lambda$.

Making use of the coordinate basis we find, for example,

$$
\begin{aligned}
& D T=D_{\mu} T^{\alpha}{ }_{\beta} E^{\mu} \otimes E_{\alpha} \otimes E^{\beta}, \\
& D_{\mu} T^{\alpha}{ }_{\beta}=\partial_{\mu} T^{\alpha}{ }_{\beta}+T^{\rho}{ }_{\beta} \Gamma^{\alpha}{ }_{\rho \mu}-T^{\alpha}{ }_{\rho} \Gamma^{\rho}{ }_{\beta \mu} \\
& \\
& +w(T) W_{\mu} T^{\alpha}{ }_{\beta} .
\end{aligned}
$$

Unlike the Weyl potential and the connection $\Gamma_{\beta \mu}^{\alpha}$, there is no unique metric on the space. Instead, the metric is taken to be of Weyl type $w(g)=2$, so that under a Weyl transformation

$$
g^{\prime}=e^{2 \Lambda(x)} g \text {. }
$$

The principle fields of the theory are related by the requirement $D g=0$. In components,

$$
\begin{aligned}
D_{\mu} g_{\alpha \beta} & =0 \\
& =\partial_{\mu} g_{\alpha \beta}-g_{\rho \beta} \Gamma_{\alpha \mu}^{\rho}-g_{\alpha \rho} \Gamma_{\beta \mu}^{\rho}+2 W_{\mu} g_{\alpha \beta},
\end{aligned}
$$

which may be solved to give

$$
\Gamma_{\beta \mu}^{\alpha}=\left\{\begin{array}{c}
\alpha \\
\beta \mu
\end{array}\right\}+\left(\delta^{\alpha}{ }_{\beta} W_{\mu}+\delta^{\alpha}{ }_{\mu} W_{\beta}-g_{\beta \mu} W^{\alpha}\right) .
$$

Vanishing torsion has been assumed in Eq. (2.7), so that

$$
\left\{\begin{array}{c}
\alpha \\
\beta \mu
\end{array}\right\}
$$

is the usual Christoffel connection. The form of the curvature tensor is given by the usual expression in terms of the full connection:

$R_{\beta \mu \nu}^{\alpha}=\Gamma_{\beta v, \mu}^{\alpha}-\Gamma_{\beta \mu, v}^{\alpha}+\Gamma_{\rho \mu}^{\alpha} \Gamma_{\beta v}^{\rho}-\Gamma_{\rho v}^{\alpha} \Gamma_{\beta \mu}^{\rho}$.

Unlike the Riemannian curvature tensor, however, the Weyl curvature has nonvanishing trace on the first pair of indices:

$$
\frac{1}{2} R_{\alpha \mu \nu}^{\alpha}=W_{v, \mu}-W_{\mu, \nu} \equiv W_{\mu \nu},
$$


$W_{\mu \nu}$ is the gauge-invariant field strength of the Weyl potential.

Finally, we define two fields to be Weyl conjugate if they have the same Lorentz transformation properties but opposite Weyl weights. ${ }^{7}$

\section{A THEORY OF MEASUREMENT FOR WEYL GEOMETRY}

Within the structure of a Weyl geometry, there has not yet been developed a complete and consistent theory of measurement. One must deal with an equivalence class of metrics rather than a single metric, and a notion of length that changes nonintegrably about the manifold. Under these circumstances, it is not immediately clear that measurement is possible. However, there is one class of objects which exists unambiguously even in a Weyl geometry-those of vanishing Weyl weight. This class will form the basis of our theory of measurement.

We begin with some elementary observations concerning fields in Weyl geometry. Fields with nonvanishing Weyl weight will experience changes upon parallel transport which depend on $W_{\mu}$. For example, the mass squared of a particle parallel transported along a path with unit tangent vector $u^{\mu}=d x^{\mu} / d \tau$ satisfies

$$
0=u^{\mu} D_{\mu}\left(m^{2}\right)=u^{\mu} \partial_{\mu}\left(m^{2}\right)+w\left(m^{2}\right) u^{\mu} W_{\mu} m^{2} .
$$

Integrating along the path of motion, we find a path dependence for the mass squared:

$$
m^{2}=m_{0}^{2} \exp \left(w\left(m^{2}\right) \int W_{\mu} u^{\mu} d \tau\right)
$$

where the line integral $\int W_{\mu} d x^{\mu}$ has been written in terms of the path parameter $\tau$. This "conformal mass" was first introduced by Schouten and Haantjes. ${ }^{8}$ It is easily seen that the dependence on the Weyl vector is necessary to ensure that $\mathrm{m}^{2}$ is independent of the gauge choice; however, the resulting dependence on $W_{\mu}$ can produce measurable mass change. At least two masses are required to make such a measurement, with one providing a standard for comparison. If two particles of identical mass are allowed to propagate freely (i.e., by parallel transport) along different paths, then brought together and the masses compared, there will be a difference given by

$$
\Delta m^{2}=m_{0}^{2} \exp \left(w\left(m^{2}\right) \oint W_{\mu} u^{\mu} d \tau\right),
$$

or

$$
\Delta m^{2}=m_{0}^{2} \exp \left[w\left(m^{2}\right) \iint_{S} W_{\mu \nu} d S^{\mu \nu}\right],
$$

where $d S^{\mu \nu}$ is an element of any two-surface $S$ bounded by the closed curve defined by the paths of the two particles. Thus, unless the surface integral over the Weyl field strength vanishes, there will be a path dependence of masses and of any other field of nonzero weight.

Equation (3.2) expresses the central difficulty of making a measurement in a general Weyl geometry. All tensor fields must be combined to form scalars to compare with measurements. For example, when we "measure" the $z$ component of a vector we actually measure the scalar formed by taking the inner product of the vector with a parallel transported unit basis vector in the $z$ direction. Moreover, all scalars vary according to an appropriate form of Eq. (3.2). Therefore, no weightful combination formed from any rank of tensor fields can be assigned an unambiguous value independent of its history in the presence of a general Weyl field.

We assume the following.

First postulate: Quantities of vanishing Weyl weight are physically meaningful ("observables").

For a field with a nontrivial Weyl weight to have any physical meaning, it must be possible to construct weightless scalars by combining it with other fields. We make the assumption that this is always possible. In fact, we will make the stronger assumption that all fields occur in Weyl-conjugate pairs, since this is the case in the conformal and superconformal groups. ${ }^{7,9}$ Then the product of a field and its Weyl conjugate always provides a measurable quantity and we are guaranteed to have measurable consequences of weightful fields. Zero-weight fields may be regarded as self-conjugate.

Second postulate: Weightful physical fields occur in conjugate pairs satisfying conjugate equations of motion.

Given the possibility of constructing weightless fields from a conjugate pair of weightful tensor fields $M=\left(M_{+}, M_{-}\right)$, we still face the difficulty of specifying how each member of the pair evolves. Part of the answer is supplied by the Weyl conjugacy. If we assume that the two fields $M_{ \pm}$evolve by parallel transport along a path with tangent $u^{\mu}$ then

$$
0=u^{\mu} D_{\mu} M_{ \pm}=u^{\mu} \bar{D}_{\mu} M_{ \pm} \pm w(M) M_{ \pm} W_{\mu} u^{\mu}
$$

where $\bar{D}_{\mu}$ is a derivation using the full connection of Eq. (2.7), and without loss of generality we set $w(M)=w\left(M_{+}\right)>0$. Since Eq. (3.5) depends explicitly on the weights $\pm w(M)$ it is conceivable that the existence of a solution to Eq. (3.5) for $\boldsymbol{M}_{+}$might preclude the simultaneous existence of a solution for $M_{-}$for the same path. However, the dependence on Weyl weight of the evolution of $M_{ \pm}$may be expressed as

$$
M_{ \pm} \equiv M \exp \left[\mp w(M) \int W_{\mu} u^{\mu} d \tau\right)
$$

where $\mathcal{M}$ is weightless and evolves according to

$$
u^{\mu} \bar{D}_{\mu} M=0 \text {. }
$$

Therefore parallel transport of $M_{ \pm}$with the derivative $D_{\mu}$ implies parallel transport of $\mathcal{M}$ with $\bar{D}_{\mu}$, and this latter transport is independent of the Weyl weight. So when $M_{+}$evolves along some path, the conjugacy of the equations of motion implies that the same motion is possible for $M_{-}$, the only difference being that while $M_{+}$ contracts according to Eq. (3.6), $M_{-}$expands. Therefore, we need only specify the evolution of one member of the pair, $M_{+}$.

Now suppose we wish to measure some characteristic of $M$. Since $M$ is weightful, it can always be scaled by an arbitrary gauge function. We correct the problem by transporting $M$ along a path so that its covariant deriva- 
tive in the direction of the motion vanishes. Then the change in size of $M$ along any trajectory in a Weyl geometry is specified by Eq. (3.6). But it is not clear that we can tell which path a particle has taken. In a Riemannian geometry there exist geodesics which are assumed to provide the paths of classical matter. This is not necessarily true in a Weyl space.

For example, it is clear that a point particle will no longer follow a path of maximal proper time since a rescaling can make any timelike path into one of maximal proper time. In Sec. V we will derive the most general case for which an extremal path can exist in a nontrivial Weyl geometry. Since this case involves a restriction of the Weyl field, for the general formulation we must assume that all paths are possible in a generic Weyl geometry.

Now we are in a position to study the motion of $M$. We begin with the observation that a Weyl geometry provides us $a$ priori with the existence of a probability: the probability $P_{A B}(M)$ of finding a value $M$ at point $B$ for a system which is known to have had a value $M_{0}$ at point $A$. Finding $P_{A B}(M)$ is tantamount to finding the fraction of paths which the system may follow which lead to any given value of $M$. That is not difficult when the possible paths of the system are limited, but in a general Weyl geometry there may be no special paths. Instead, we may have to settle for moments of the distribution-the mean, the standard deviation and so forth.

To find the average value of magnitude of $M$, denoted by $\langle M\rangle$, we integrate Eq. (3.6) over all paths

$$
\langle M\rangle=\int \mathcal{D}[x] M_{0} \exp \left[w(M) \int_{A}^{B} W_{\mu} u^{\mu} d \tau\right),
$$

where the usual path-integral normalization is included implicitly in $\mathscr{D}[x]$. The meaning here is clear enough in principle. We calculate the change in size along several paths and average them. Then we do it again but with a larger selection of paths. Continuing in this way we are led to the average in the limits as all paths are included. In practice of course this is an uncountably infinite limiting problem requiring special techniques, since the space of paths is larger than the space of real numbers.

Equation (3.8) tells us that if the system arrives at point $B$, we can predict the probability of measuring any particular magnitude for $\boldsymbol{M}$. However, it provides us no clue as to whether we should expect $M$ to actually reach $B$. We have assumed that $M$ might evolve along any path, but we need some idea which paths are likely.

To motivate the answer, we must explore the meaning of a path average. Consider a simple special case in which the relevant paths can be parametrized by a single variable $x$ and the weighting functional $f[x]$ is simply a function $f(x)$ :

$$
\langle f\rangle=\int f(x) \mathscr{D}[x] .
$$

The integral is just a path-weighted average of $f(x)$ over all values of $x$, but the $D x$ brings in a normalization which may be divergent. The path integral may be rewritten as a normal integral of the relative probability $\rho(u)$ of a certain value $u=f(x)$ occurring, times the value $u$ :

$$
G=\int u \rho(u) d u
$$

The density $\rho$ depends on how often a particular value of $f(x)$ occurs. As shown rigorously in the Appendix, in a small neighborhood of a path $x_{0}$ the number of paths for which $f(x)$ takes on values near $f\left(x_{0}\right)$ is proportional to

$$
\rho(u) d u \sim \frac{1}{f^{\prime}\left(x_{0}\right)} d u
$$

so that $\rho$ is singular whenever $f^{\prime}(x)=0$. For such singular instances of $\rho, G$ will generally only include contributions from the zeros of $f^{\prime}(x)$. Whenever the integral of $\rho(u)$ over a singularity $f^{\prime}\left(x_{0}\right)=0$ is itself singular the normalization of the whole path integral will be infinite, overwhelming the contributions from other paths while giving a finite contribution at $x_{0}$.

To produce a distribution which is equally weighted, so that each value of $u$ gives the same contribution to the average, simply requires a weighting function $f(x)$ satisfying

$$
u \rho(u) \sim \frac{f(x)}{f^{\prime}(x)}=\lambda^{-1}=\text { const }
$$

so that

$$
f(x)=A e^{\lambda x} .
$$

If we replace $x$ in Eq. (3.13) with a functional $g[x]$ then the path integral of $f(g[x])$ will be uniformly sensitive to changes in $g[x]$ without being affected by the particular magnitude of $g[x]$ :

$$
u \rho(u) \sim \frac{1}{\lambda \delta g[x]} .
$$

This effect is seen in a Wiener integral ${ }^{10}$ where the weighting functional $e^{S}$ is an exponential. Changes in the action are equally weighted and the overall magnitude of the action is irrelevant. A moment's thought shows why this is so. As the value of $S$ goes to $-\infty, \rho(u)$ becomes singular since there is a zero of $e^{S} \delta S$. But these contributions are exponentially damped by the $u=e^{S}$ in the numerator. Similarly, when $S$ becomes large there is a compensating $e^{S}$ in the denominator. Therefore, significant contributions to the Wiener integral occur only when $S$ is extremal.

We now combine these observations with the following postulate.

Third postulate: The probability that a system will undergo a given infinitesimal displacement $d x^{\mu}$ is inversely proportional to the change in length such a displacement produces in the system.

The incremental change in the length $l$ characterizing a system of weight $w(M)$ is given by

$$
d l=w(M) W_{\mu} d x^{\mu}=w(M) W_{\mu} u^{\mu} d \tau
$$

for a displacement of $d x^{\mu}$. We want $1 / d l$ to play the role of $u \rho(u)$ in the simplified example above so we set $f=e^{l}$. Then the probability of the system reaching any spacetime point $x$ from $x_{0}$ is

$$
G\left(x_{0} ; x\right)=\int \mathscr{D}[x] e^{w(M)} \int_{x_{0}}^{x} W_{\mu} u^{\mu} d \tau
$$


We note that the existence of the path integral of Eq. (3.16) is guaranteed. ${ }^{10}$ One further step is now required. Comparison of Eq. (3.16) with (3.8), or a direct check, shows that Eq. (3.16) is gauge dependent. One advantage of the path formulation, however, is that such a gauge transformation only depends on the end points $x_{0}$ and $x$ and therefore comes out of the path integral:

$$
\begin{aligned}
G^{\prime}\left(x_{0} ; x\right) \\
\quad=\int \mathscr{D}[x] \exp \left(w(M) \int_{x_{0}}^{x}\left(W_{\mu}-\partial_{\mu} \phi\right) u^{\mu} d \tau\right) \\
\quad=\int \mathscr{D}[x] e^{-w(M)\left[\phi(x)-\phi\left(x_{0}\right)\right]} \exp \left(w(M) \int_{x_{0}}^{x} W_{\mu} u^{\mu} d \tau\right) \\
\quad=e^{-w(M)\left[\phi(x)-\phi\left(x_{0}\right)\right]} \int \mathcal{D}[x] \exp \left(w(M) \int_{x_{0}}^{x} W_{\mu} u^{\mu} d \tau\right) .
\end{aligned}
$$

This means that we can eliminate the gauge factor by multiplying by the Weyl conjugate expression,

$$
\begin{aligned}
& \bar{G}^{\prime}\left(x_{0} ; x\right) \\
& \quad=e^{w(M)\left[\phi(x)-\phi\left(x_{0}\right)\right]} \int \mathcal{D}[x] \exp \left(-w(M) \int_{x_{0}}^{x} W_{\mu} u^{\mu} d \tau\right),
\end{aligned}
$$

to give a physically meaningful gauge-invariant probability:

$$
P\left(x_{0}, x\right)=\bar{G}\left(x_{0} ; x\right) G\left(x_{0} ; x\right) .
$$

$P\left(x_{0}, x\right)$ is the probability of detecting the dilating system at $x$, given its presence at $x_{0}$. It may be thought of as the joint probability of finding both $M$ and $\bar{M}$ at $x$. Because of their gauge dependence neither probability is directly meaningful in itself, but the joint probability is physical.

It is important to note that Eq. (3.16) involves a real path integral, unlike the phase integral of quantum theory. This corresponds to the difference between Weyl's original gauge theory, and later unitary gauge theories. Here the phase invariance of a wave function, $\psi^{\prime}=e^{i \phi} \psi$, is replaced by conformal invariance, $M^{\prime}=e^{\phi} M$. This is the same factor of $i$ discovered by London in 1927. Since London's time, gauge transformations have always appeared as phases and the wave interpretation has been maintained. What is intended here is just the opposite: we will maintain a real gauge transformation and change our interpretation of physical phenomena.

Observe the similarity between the probability of $M$ arriving at $x$, Eq. (3.16), and the average value of $M$ at $B$, Eq. (3.8). If the gauge is fixed everywhere so that $M_{0}=1$ the expressions become identical. This again represents the London result: London showed that the complexvalued length dilation factor $e^{i \int W}$ is proportional to the complex-valued Schrödinger wave function. We have shown that the average value of the real-valued dilation factor is proportional to the real-valued probability amplitude for position.

Finally, we note a possible alternative formulation. The gauge-invariant probability, Eq. (3.19), is built as a product of path integrals. Gauge invariance could also be achieved by combining Weyl conjugate pairs in a single path integral, where the quantity to be averaged is the exponential of the Weyl vector for one field integrated along one path times the exponential of the conjugate field integrated along a different path. Because of the change in sign from the conjugacy the pair of integrals combines to form a single integral of $W_{\mu}$ over a closed loop. Applying Stokes's theorem the gauge invariance of the expression is manifest.

In this latter formulation it is not even necessary to retain the initial and final points of integration since the integral of the Weyl field is gauge invariant for any loop. Thus, one may specify initial and final loop configurations and average over connecting world sheets in order to make a gauge-invariant prediction. This may be a natural formulation in the sense that the initial and final states correspond to flux integrals at the initial and final times. Indeed, the measurement of a field at a point is an idealization and it may be more realistic to regard the initial data set as a measurement of flux.

\section{QUANTUM MECHANICS FROM WEYL GEOMETRY}

In the remainder of this paper we will explore the consequences of our original formulation, Eqs. (3.16) and (3.19). We begin by showing the equivalence to quantum mechanics of the nonrelativistic limit of Eq. (3.16) when the exponent in the path integral is identified with a multiple of the classical action:

$$
\int W_{\mu} u^{\mu} d \tau=\lambda S=\lambda \int L d \tau
$$

Later Eq. (4.1) will provide us with the key to identifying preferred paths. The integrands in Eq. (4.1) may also be equated, except for the possible addition of the total derivative of a function of $\tau$. But such a derivative is already known to be both a gauge freedom of $W_{\mu}$ and a transformation of $L$ that leaves the equations of motion unaltered. So the possible equivalent versions of $L$ may be understood as gauge changes of the underlying geometry. This identification fixes the physical interpretation of $W_{\mu}$, up to the gauge choice. Since $u^{\mu}=\dot{x}^{\mu}$, equating the integrands gives

$$
\lambda P_{\mu} \equiv \lambda \frac{\partial L}{\partial u^{\mu}}=W_{\mu},
$$

so that $W_{\mu}$ is proportional to the generalized momentum $P_{\mu}$ conjugate to $x^{\mu}$. Notice the difference between the identification of $W_{\mu}$ as the conjugate momentum and Weyl's original identification of $W_{\mu}$ with the electromagnetic potential $Q A_{\mu}$. In the corresponding case, our approach gives

$$
W_{\mu}=\lambda\left(p_{\mu}+Q A_{\mu}\right)
$$

so that all energy provides a source of expansion rather than just electromagnetic energy. We will see in Sec. V that this makes a crucial difference for the classical interpretation. Note also that this new interpretation of the Weyl vector still allows gauge transformations of $W_{\mu}$ to 
be identified with gauge transformations of $\boldsymbol{A}_{\mu}$.

We now move to the nonrelativistic limit of the path integral to find a differential equation for the amplitudes $G\left(x_{0}, x\right)$. It is convenient to explicitly separate the kinetic term $p_{\mu} u^{\mu}$ from $W_{\mu} u^{\mu}$. This enables us to immediately identify the path integral of Eq. (3.16) with a Wiener path integral. With full generality we may write

$$
W_{\mu} \equiv \lambda\left(p_{\mu}+\widetilde{W}_{\mu}\right),
$$

where any gauge transformation is understood to apply to $\widetilde{W}_{\mu}$. Now consider the nonrelativistic limit. The integral $\int p_{\mu} u^{\mu} d \tau$ may be written as $m c^{2} \int d \tau$, which we may approximate as the integral of the rest energy minus the classical kinetic term:

$$
m c^{2} \int d \tau \approx \int\left(m c^{2}-\frac{m}{2} \mathbf{v}^{2}\right) d t
$$

To this order the path integral becomes

$$
\begin{gathered}
G\left(x_{0} ; x\right)=\int \mathcal{D}[x] \exp \left[\lambda w ( M ) \left[\int \left(\frac{1}{2} m \mathbf{v}^{2}+\tilde{\mathbf{w}} \cdot \mathbf{v}-\tilde{W}^{0}\right.\right.\right. \\
\left.\left.\left.-m c^{2}\right) d t\right]\right]
\end{gathered}
$$

where the limits of integration have been suppressed. This is precisely of the form

$$
\left.P\left(x_{0} ; x\right)=\int \mathscr{D}[x] \exp \left(-\frac{1}{2} \int\left[(\dot{\mathbf{q}}+\mathbf{h})^{2}-\nabla \cdot \mathbf{h}\right] d t\right)\right],
$$

where $P\left(x_{0} ; x\right)$ is the propagator for the Fokker-Planck equation, ${ }^{11,12}$

$$
\frac{\partial P}{\partial t}=\frac{1}{2} \nabla^{2} P+\nabla \cdot(\mathbf{h} P),
$$

provided we make the identifications

$$
\begin{aligned}
& \dot{\mathbf{q}}=\sqrt{-w(M) \lambda m} \mathbf{v}, \quad \nabla_{\mathbf{x}}=\sqrt{-w(M) \lambda m} \nabla_{q}, \\
& \psi=P e^{-2 m c^{2}}, \quad \mathbf{h}=\sqrt{-w(M) \lambda / m} \tilde{\mathbf{W}}, \\
& 2 w(M) \lambda\left(m c^{2}+\widetilde{\mathbf{W}}^{0}\right)=\mathbf{h}^{2}-\nabla \cdot \mathbf{h} .
\end{aligned}
$$

Carrying out the substitutions and setting $\lambda \widetilde{\mathbf{W}}_{\mu}=-Q(\lambda \varphi, \mathbf{A})$, we find that

$$
\psi(x)=\int \psi\left(x^{\prime}\right) G\left(x, x^{\prime}\right) d x^{\prime}
$$

is a solution to

$$
\begin{aligned}
\frac{1}{w(M) \lambda} \frac{\partial \psi}{\partial t}= & -\frac{1}{2 m[w(M) \lambda]^{2}}[\nabla+w(M) Q \mathbf{A}]^{2} \psi \\
& +\left(m c^{2}+Q \varphi\right) \psi
\end{aligned}
$$

with initial condition $\psi=\psi\left(x^{\prime}\right)$. If we set $\lambda=\hbar^{-1}$ and the time is allowed to become imaginary, Eq. (4.11) gives precisely the Schrödinger equation minimally coupled to electromagnetism. We will choose $\lambda=\hbar^{-1}$, but introducing imaginary time would be arbitrary and is not necessary. In keeping with our program, we will interpret Eq. (4.11) directly, as a stochastic form of quantum mechanics. ${ }^{13}$ Evidently the Weyl weight serves the function of $i$, changing sign appropriately for the conjugate field.

The emergence of the Fokker-Planck equation shows clearly that we are dealing with a diffusion process. In Ref. 13, Nelson shows that the Schrödinger equation is rigorously equivalent to such a diffusion process and that phenomena such as single- and double-slit interference and nodes of the wave function are correctly predicted from that point of view. The odd fact that diffusion can account for nodes and interference prompts that author to write "If you are familiar with dissipative diffusion, forget what you know-it has no more connection with conservative diffusion than has the fall of a sponge with celestial mechanics." Explicit calculations show these phenomena in detail. Additionally, Nelson shows that the exclusion principle and spin have a natural place in a stochastic model. The only difficulty encountered by stochastic mechanics arises from its claim that the stochastic particle is "physically real." Even with this claim, agreement with Bell's theorem and experiment can be maintined by using non-Markovian diffusion. However, the present formulation in the context of Weyl geometry avoids the locality problem altogether, since the path of the particle between measurements is not a gaugeinvariant concept. Just as in quantum mechanics, the Weyl geometric approach to quantization claims that a dimensionful field has no definite measurable reality. Only zero-weight combinations of such fields are physical.

As a simple example of the equivalence between the stochastic model and standard quantum mechanics, and to illustrate the Weyl interpretation, we note that the stationary state solutions for $\psi(x)$ are identical to the corresponding solutions to the Schrödinger equation provided only that we assume a time dependence of

$$
\psi(x, t)=\psi(x) e^{\lambda w(M) E t} .
$$

Where an energy quantum number occurs in the solution of the Schrödinger equation, identical energy quantum numbers will also occur.

It remains only to interpret the meaning of the exponential time dependence, which is no longer the oscillating solution of wave mechanics. The interpretation, however, is immediate since we are studying systems which expand and contract. The exponential merely represents the time development of a system which expands at a rate $\hbar^{-1} E$. Since the theory is scale invariant, such expansion is not directly physically observable. The only meaningful magnitudes are those which can be measured, such as the values of $E$ for which the equation has solutions and the weightless probabilities constructed from $\psi$ and its Weyl conjugate. The conjugate of an expanding system is a contracting one, and when an invariant is formed of the pair, the exponential dependence will vanish.

Finally, we observe that while the wave picture explains some phenomena such as interference more readily than the stochastic picture, other results follow more easily from the point of Weyl geometry. The simplest example is that of a free wave packet, which is a spreading Gaussian with a decaying amplitude. In quantum mechanics such way packets must be constructed from plane-wave solutions to the Schrödinger equation by su- 
perposition. But the Weyl picture leads us to expect expansion of the field, and the diffusion equation leads us to the Gaussian form directly.

\section{CLASSICAL LIMIT OF WEYL GEOMETRY}

\section{A. The form of the Weyl vector in the classical limit}

Based on the results of Sec. IV we can now identify the exponent in the Wiener integral with the classical action in units of the Planck constant:

$$
\frac{1}{\hbar} S=\int W_{\mu} u^{\mu} d \tau
$$

A classical limit of the Weyl geometry will exist whenever there is an extremum to the action, just as occurs for the Feynman path integral. The argument that this is true for the Feynman integral is based on the idea that far from an extremum of the action the exponential, $e^{i S}$, oscillates rapidly. That the same result holds for the Wiener integral follows from our construction of Sec. III, which shows that the exponential weighting produces a result that depends only on changes in the exponent.

Thus, a classical limit of Eq. (3.16) occurs if and only if

$$
\Psi \equiv \exp \left(w(M) \int_{x_{0}}^{x} W_{\mu} u^{\mu} d \tau\right)
$$

is extremal. When $\delta \Psi=0$ the physical paths in a Weyl geometry are those of extremal length just as in a Riemannian geometry. There is a difference here, however, in that extremal length refers to both the usual line element $d s^{2}$, and to the length factor $\Psi$.

Next, we show that $\delta \Psi=0$ corresponds to a special case of the Weyl field. If the variation of a generic function $\Psi$ could always be made extremal for some path then the Wiener integral would always reduce to a sum over classical paths. Instead, we will see that for arbitrary paths the variation $\delta \Psi$ vanishes only when the Weyl field is of a particular form.

Carrying out the variation $\delta x^{\mu}$ we require

$$
\int_{A}^{B} d \tau\left(W_{\mu, v}-W_{v, \mu}\right) u^{\mu} \delta x^{v}=0
$$

or

$$
W_{\mu} u^{\mu}=0 \text {. }
$$

Generalizing a similar calculation applied by $\operatorname{Dirac}^{14}$ to the motion of a charged particle, we show that Eq. (5.4) restricts the form of $W_{\mu \nu}$. It follows from Eq. (5.4) that there exists a vector field $v^{v}$ such that

$$
\frac{1}{2} \epsilon^{\mu v \alpha \beta} W_{\alpha \beta}=u^{\mu} v^{v}-u^{v} v^{\mu},
$$

where $\epsilon^{\mu v \alpha \beta}$ is the Levi-Civita tensor. In addition, there is a Bianchi identity for $W_{\mu \nu}$ :

$$
\epsilon^{\alpha \beta \sigma \rho} D_{\sigma} W_{\alpha \beta}=0 \text {. }
$$

Combining Eqs. (5.5) and (5.6),

$$
\begin{aligned}
0 & =D_{v}\left(u^{\mu} v^{v}-u^{v} v^{\mu}\right) \\
& =v^{v} D_{v} u^{\mu}-u^{v} D_{v} v^{\mu}+\left(D_{v} v^{v}\right) u^{\mu}-\left(D_{v} u^{v}\right) v^{\mu} .
\end{aligned}
$$

The first two terms on the right-hand side define the commutator of the two vector fields $u^{\mu}$ and $v^{\mu}$ (Ref. 15) and the resulting identity

$$
[u, v]^{\mu}=\left(D_{v} v^{v}\right) u^{\mu}-\left(D_{v} u^{v}\right) v^{\mu}
$$

giving the commutator of two vector fields as a linear combination of the same two vector fields says that the set $\left\{u^{\mu}, v^{v}\right\}$ is involute. This is precisely the condition required by the Frobenius theorem in order for $u^{\mu}$ and $v^{\mu}$ to be tangent to an integrable two-dimensional submanifold of spacetime. Again following Dirac we let $\chi$ and $\xi$ be two functions which are constant on this surface. Then

$\left(D_{\mu} \chi\right) u^{\mu}=\left(D_{\mu} \chi\right) v^{\mu}=\left(D_{\mu} \xi\right) u^{\mu}=\left(D_{\mu} \xi\right) v^{\mu}=0$.

Equation (5.5) then shows

$$
\epsilon^{\mu v \alpha \beta} W_{\alpha \beta} D_{\mu} \chi=\epsilon^{\mu v \alpha \beta} W_{\alpha \beta} D_{\mu} \xi=0,
$$

resulting in

$$
W_{\alpha \beta}=\alpha\left(D_{\alpha} \chi D_{\beta} \xi-D_{\beta} \chi D_{\alpha} \xi\right) .
$$

The Bianchi identity applied to Eq. (5.11) then implies that $\alpha$ is a function of $\xi$ and $\chi$ only and so may be set to unity by a reparametrization. Renaming $\chi$ and $\xi$ to absorb $\alpha$, we conclude that

$$
W_{\alpha \beta}=D_{\alpha} \chi D_{\beta} \xi-D_{\beta} \chi D_{\alpha} \xi=\partial_{\alpha} \chi \partial_{\beta} \xi-\partial_{\beta} \chi \partial_{\alpha} \xi
$$

and up to a gauge transformation $W_{\alpha}$ may be written as

$$
W_{\alpha}=\xi \partial_{\alpha} \chi
$$

for any appropriately normalized functions $\xi, \chi$ satisfying Eqs. (5.9).

Equation (5.13) is a rather remarkable relation. Note that it represents a restricted form of the vector field $W^{a}$ since it is easy to find a Weyl vector such that $W_{\mu v} u^{v} \sim W_{\mu 0} \neq 0$ for all nonspacelike $u^{v}$. Since Eq. (5.13) follows for an arbitrary set of paths $u^{\alpha}$, it is clear that not all Weyl fields will have a classical limit. Therefore, according to the arguments at the beginning of this section, the generic Weyl geometry lacks preferred paths and requires a path average. Such a Weyl field has an action with no extremum, in direct contrast with our usual assumptions.

The existence of action functionals without extrema raises the question of whether there exists a valid perturbation theory for the nonclassical fields. Certainly it is valid to write a generic vector $W_{\mu}$ in the form

$$
W_{\mu}=\partial_{\mu} \phi+\xi \partial_{\mu} \chi+R_{\mu}
$$

and expand for small $R_{\mu}$. But there is no assurance that the remainder $R_{\mu}$ will be small.

We now return to the restricted form for the classical limit of the Weyl field given in Eq. (5.13). Several issues raised by this form are discussed in subsections B-D below.

\section{B. Classical size change}

Since Eq. (5.13) implies a nontrivial Weyl field, $W_{\mu \nu} \neq 0$, we must explore the meaning of the resulting 
changes in the sizes of dimensionful quantities as they move about in spacetime. While it is true that $W_{\mu \nu} \neq 0$ means that for certain motions the sizes of weightful quantities will change as they move, in the classical limit bodies are restricted to move along special paths. Moreover, Eqs. (5.9) and (5.13) ensure that a gauge exists where

$$
W_{\alpha} u^{\alpha}=0
$$

which means that in that gauge in the classical limit, weightful bodies follow the preferred classical trajectories and therefore experience no dilation.

This is a somewhat surprising result. Normally we imagine that any spacetime path may be followed and consequently any component of a field may be measured. But it is clearly impossible to actually follow every "possible" classical path. As a result, the size changes associated with nonvanishing Weyl field strength are not classically observable. It is easy to find examples of sets of paths which exclude the possibility of detecting size changes. The simplest case is to consider a parallel congruence of straight lines. Choose a Weyl field such that only for motions perpendicular to the congruence do objects change in size. Now restrict the allowed paths of objects to lie along the congruence. Clearly, for such motions size changes never occur. Then the size changes are unobservable.

Neither will it do to suppose that slightly changing the set of actual paths will allow detection of the size change. Changing the preferred paths implies changing the Weyl field in just such a way as to mask the size change. This effect may be thought of in terms of the absence of ideal test particles. Without idealized test particles in general relativity, flat spacetime is unobservable because all matter causes curvature. In the present interpretation of Weyl geometry the coupling of matter to the geometry is much stronger than in general relativity so that the flow of matter cannot be altered without altering the direction of the geodesics correspondingly.

A dramatic example of the test particle problem is provided by the motions of charged particles (see Sec. V D below). Quite distinct motions can follow from seemingly similar initial conditions. If we give an electron and a positron the same initial position and velocity in an external field they will follow different paths. This is because the change of charge dramatically alters the geometryyou cannot make infinitesimally small particles of charge, and introducing a (small) positive charge in place of a (small) negative one means the local geometry is now expanding (rapidly) where it had been contracting (rapidly). Since the particle responds only to the local geometry, swapping charges drastically changes the experiment.

Finally, note that the unobservability of size changes of weightful quantities explains why classical physics contains more observables than quantum physics. In the view of Sec. III, only one physical quantity is available for each conjugate pair of observables. But in the classical limit where the size changes are unobservable both conjugate partners may be assigned unambiguous values.

\section{Agreement with axiomatic approaches to general relativity}

There have been a number of attempts to derive the properties of Riemannian geometry from locally observable properties of the world. Perhaps the most successful effort in this direction is the work of Ehlers, Pirani, and Schild $^{16}$ who show that the null geodesics of the local conformal structure implicit in the paths of light rays agree with the null limit of the geodesics of the local projective structure implied by the paths of massive particles only if the world is described by a Weyl geometry. They further restrict spacetime to be Riemannian by the additional assumption that the rates of clocks are independent of their histories. This assumption is less evident than the others. Indeed, Audretsch ${ }^{5}$ dismisses the extra assumption as being "unsatisfactory from the point of view of an axiomatic scheme" and concludes ". . . there have been several efforts after 1970 to describe an alternative constructive approach to general relativity based on more primitive concepts. All these different approaches end up with assigning to space-time a Weyl geometry instead of the further restricted Riemann geometry of general relativity."

Audretsch, and later Audretsch, Gähler and Straumann, ${ }^{6}$ tried to restrict the axiomatic geometry to be Riemannian in another way. They considered the paths of quantum fields in the classical limit. They claim that in the $\hbar \rightarrow 0$ limit the Dirac current follows the same set of conformal and projective geodesics as classical matter only if the Weyl field strength $W_{\alpha \beta}$ vanishes. However, their argument overlooks an important possibility.

Briefly, the authors use a WKB approximation of the Dirac four-current, $J_{\alpha}$, to derive its directional Weyl derivative [Eq. (6.10) of Ref. 5]

$$
J^{\alpha} D_{\alpha} J_{\beta}=J^{\alpha} D_{\alpha}\left(\ln \frac{1}{m} \sqrt{J_{\rho} J^{\rho}}\right) J_{\beta}+J_{\rho} J^{\rho} D_{\beta}(\ln m),
$$

where $J_{\alpha}$ is of the approximate WKB form $J_{\alpha}=\eta \partial_{\alpha} S$ for a certain function $\eta$. Because the right-hand side of Eq. (5.16) is not, in general, proportional to $J_{\beta}$, the current path is not autoparallel. The authors conclude that requiring Eq. (5.16) to be the equation of a geodesic forces the last term to vanish:

$$
D_{\beta} m=\partial_{\beta} m-\frac{1}{2} m W_{\beta}=0 \text {. }
$$

Then

$$
W_{\beta}=\partial_{\beta} \ln \left(m^{2}\right), \quad W_{\alpha \beta}=0
$$

and the spacetime becomes Riemannian. The oversight is that for Eq. (5.16) to be the equation of a geodesic requires only that the final term be proportional to the current $J_{\beta}$ :

$$
D_{\beta}(\ln m)=\alpha J_{\beta}=\eta^{\prime} \partial_{\beta} S,
$$

where $\alpha$ is an arbitrary function and $\eta^{\prime}=\alpha \eta$. The resulting form for the Weyl field is

$$
W_{\beta}=-2 \eta^{\prime} \partial_{\beta} S+\partial_{\beta} \ln \left(m^{2}\right) \text {. }
$$


Rather than a contradiction there is a striking confirmation of the form for $W_{\alpha}$ given in Eq. (5.13). Even the orthogonality condition, Eq. (5.9), is satisfied because $S$ in Eq. (5.20) is the action evaluated along the classical path. The derivative of this action along the path vanishes, but other directional derivatives need not.

\section{Observable consequences of the classical Weyl field}

As described in Sec. V B, size changes induced by a classical Weyl field are unobservable. However, the Weyl field itself must be present and consequently must have been detected. Finding the physical field that it corresponds to simply requires substituting the appropriate conjugate momentum for $W_{\mu}$ in the classical equation of motion $W_{\mu v} u^{v}=0$. Since the only long-range forces are gravity and electromagnetism, and gravity is still accounted for by the Riemannian curvature, $W_{\mu}$ must be electromagnetic. The most general classical conjugate momentum is therefore that of a point particle with charge $q$ moving in an electromagnetic field. In an arbitrary gauge

$$
W_{\mu}=\frac{1}{\hbar}\left(p_{\mu}+q A_{\mu}+\partial_{\mu} \Lambda\right),
$$

where $p_{\mu}=m u_{\mu}$ and $u_{\mu} u^{\mu}=-1$. Then

$$
\begin{aligned}
& 0=W_{\mu \nu} u^{v}=\frac{1}{\hbar}\left(p_{\mu, v}-p_{v, \mu}+q A_{\mu, v}-q A_{v, \mu}\right) u^{v} \\
& \text { or, using }\left(u_{\mu} u_{, \nu}^{\mu}=0,\right. \\
& \frac{d p^{\mu}}{d \tau}=q u_{v} F^{\mu v},
\end{aligned}
$$

the Lorentz force law. Notice that Planck's constant drops out of the equation of motion. We have taken $u^{\alpha}$ to describe a vector field in order to take the derivatives, but the final result may be construed to depend only on a single path. In a field formulation of Weyl geometry, $p^{\mu}$ will be given as a spatial integral of the stress-energy tensor.

For the interpretation of $W_{\mu}$ itself, we can combine Eq. (5.12) with the curl of Eq. (5.21) to write

$$
\partial_{\alpha} \chi \partial_{\beta} \xi-\partial_{\beta} \chi \partial_{\alpha} \xi=\frac{1}{\hbar}\left(p_{\alpha, \beta}-p_{\beta, \alpha}+q A_{\alpha, \beta}-q A_{\beta, \alpha}\right)
$$

the time component of which again gives the Lorentz force law. The spatial components may be solved for the magnetic field:

$$
\mathbf{B}=\frac{\hbar}{q}(\nabla \chi \times \nabla \xi)-\frac{m}{q}(\nabla \times \mathbf{v}) .
$$

The two fields $\chi$ and $\xi$ on the right-hand side of Eq. (5.25) are sufficient to guarantee the existence of any type of physical magnetic field. ${ }^{14}$ Conversely, we may use Eq. (5.25) to solve for the Weyl field in terms of $\mathbf{B}$ and $\mathbf{v}$, which of course depends on $\hbar$. It is perhaps interesting that for vanishing Weyl field Eq. (5.25) reduces to the London equation for superconductivity. This means that matter fields which conspire to produce a Riemannian geometry become superconducting.

These results are in sharp contrast to the predictions of the original Weyl theory, which by identifying $W_{\mu}$ with $A_{\mu}$ allows nontrivial electromagnetic fields only in the presence of observable size changes.

In the present theory, the classical limit of the Weyl field is identified with the conjugate momentum so, for a single charged particle, $W_{\mu}=p_{\mu}+q A_{\mu}$. Gauge transformations of $W_{\mu}$ are still associated with $A_{\mu}$, and the Lorentz force law with arbitrary electromagnetic fields follows from the classical form allowed for $W_{\mu}$. This special form of $W_{\mu}$ is just that required to guarantee that particles obeying the Lorentz force law will experience no measurable size change.

\section{SUMMARY}

Based on the following postulates, we have proposed a model for the interpretation of spacetime as a Weyl geometry.

(1) Quantities of vanishing Weyl weight are physically meaningful.

(2) Weightful physical fields occur in conjugate pairs satisfying conjugate equations of motion.

(3) The probability that a system will follow any given infinitesimal displacement $d x^{\mu}$ is inversely proportional to the change in length the displacement produces in the system.

From these assumptions it is argued that meaningful physical measurement in a Weyl geometry is naturally formulated by calculating the Weyl-conjugate gaugedependent probabilities for the detection of conjugate objects of Weyl weights $\pm w(M)$,

$$
\begin{aligned}
& G\left(x_{0} ; x\right)=\int \mathcal{D}[x] \exp \left(w(M) \int_{x_{0}}^{x} W_{\mu} u^{\mu} d \tau\right), \\
& \bar{G}\left(x_{0} ; x\right)=\int \mathscr{D}[x] \exp \left(-w(M) \int_{x_{0}}^{x} W_{\mu} u^{\mu} d \tau\right)
\end{aligned}
$$

and forming the gauge-independent joint probability

$$
P\left(x_{0}, x\right)=\bar{G}\left(x_{0} ; x\right) G\left(x_{0} ; x\right) .
$$

In a nonrelativistic limit, we express $G\left(x_{0} ; x\right)$ as a Wiener integral which is the propagator for the FokkerPlanck equation. This diffusion equation has solutions identical to the solutions to the minimally coupled Schrödinger equation provided the line integral of the Weyl field is identified with the action functional divided by $\hbar$ :

$$
\int W_{\mu} u^{\mu} d \tau=\frac{S}{\hbar}
$$

With this identification of $W_{\mu}$, all of the standard results of quantum mechanics including interferences, nodes, and the exclusion principle can be shown to follow. ${ }^{13}$ The stationary solutions to the diffusion equation have time dependence appropriate to expanding and contracting states, rather than the oscillating states of standard quantum theory. These dilations are not directly measur- 
able within the theory, though the eigenvalues for the rates of expansions and contractions are measurable, and given by

$$
\frac{E}{\hbar}
$$

where $E$ is an allowed energy of the system. The requirements of Bell's theorem and experiment are satisfied by the gauge dependence of the path of the system between measurements.

It is argued that the solutions of the path-integral formulation may be approximated macroscopically by extrema of the action. We prove that the most general form of the Weyl field $W_{\alpha}$ consistent with such an extremum is restricted to be of the form

$$
W_{\alpha}=\xi \partial_{\alpha} \chi,
$$

where the motion of the system is orthogonal to the gradients of the functions $\chi$ and $\xi$. Since this is not the generic form of the Weyl field, there exist Weyl fields which permit no extrema for the action. In such fields there is no preferred path and some averaging technique such as a path integral is required to make predictions of motion. For these averaged motions measurable length changes are unavoidable. It is these length changes that correspond to truly quantum behavior.

Within the macroscopic approximation above, the orthogonality condition means that particles obeying the equations of motion for extremal Weyl fields experience no dilatation. Furthermore, idealized test particles which can probe the Weyl field without altering its geodesics do not exist because the coupling of matter to expansion is so strong. This means that size changes due to extremal Weyl fields are unobservable, and it becomes possible to meaningfully measure both fields of a conjugate pair.

Even though dilations are unobservable in the classical limit, the presence of a classical Weyl vector with nonvanishing field strength is detectable. The field strength is shown to have a classical interpretation with spatial components given by

$$
(\nabla \chi \times \nabla \xi)=\frac{m}{\hbar}\left[\nabla \times \mathbf{v}+\frac{q \mathbf{B}}{m}\right) .
$$

Finally, we show that the $\hbar \rightarrow 0$ limit of the WKB approximation for the current of the Dirac field approaches the restricted classical form of the Weyl vector given above.

\section{ACKNOWLEDGMENTS}

The author appreciates stimulating discussions with Dave Hochberg during the final stages of this work.

\section{APPENDIX: THE DENSITY OF VALUES IN THE RANGE OF A FUNCTIONAL}

We wish to write the path integral of a functional $f[x]$ as a normal integral over the real-valued range of the functional $u \in\left[f_{\min }, f_{\max }\right]$,

$$
\int f[x] \mathcal{D}[x]=\int u \rho(u) d u,
$$

where the distribution $\rho(u) d u$ gives the fraction of paths $x$ for which the value of $f[x]$ lies in the interval $[u, u+d u] . \rho$ is normalized so that

$$
\int \rho(u)=1 \text {. }
$$

Only the special case where the paths $[x(\tau)]$ can be labeled by a single parameter $x$ will be considered in full. The general case is not substantially different, but we wish to avoid here the development of the necessary properties of the Lesbesgue measure on the space of paths. Nonetheless, language appropriate to the general case is used whenever possible and a sketch of the argument for a functional is given at the end.

Divide the range of $f[x]=f(x)$ into $n$ equal intervals

$$
\epsilon_{n} \equiv \frac{1}{n}\left(f_{\max }-f_{\min }\right)
$$

with midpoints

$$
f_{k}=k \epsilon_{n}+\frac{\epsilon_{n}}{2}, \quad k=0,2, \ldots, n-1 .
$$

We will assume that $f(x)$ is monotonic throughout this range. The case for general $f(x)$ is then an average of monotonic regions weighted by the path-space measure of each region. Next, construct the set of paths which lead to values of $f[x]$ close to $f_{k}$ :

$$
s_{n, k} \equiv\left\{x||\left(f[x]-f_{k}\right) \mid \leq \frac{\epsilon_{n}}{2}\right\} .
$$

A measure $\mu\left(s_{n, k}\right)$ of the set $s_{n, k}$ is proportional to $\rho\left(f_{k}\right)$ in the limit as $n \rightarrow \infty$. For the simplified case we have

$$
\rho(u) d u=A \lim _{n \rightarrow \infty} \mu\left(s_{n, k}\right)
$$

with

$$
u=\lim _{n \rightarrow \infty}\left(u_{n, k}\right)=\lim _{n \rightarrow \infty}\left(k \epsilon_{n}+\frac{\epsilon_{n}}{2}\right) .
$$

The sequence of $k$ 's is chosen in such a way that $u_{n, k}$ uniformly approaches the desired value $u$. For example, $n$ may be taken to double at each iteration and $k$ may be taken as $n$ times the first $n$ terms of the binary decimal expansion of $u / n \epsilon_{n}$. The normalization constant $A$ is just

$$
A=[\mu(\text { all paths })]^{-1}=\left(x_{\max }-x_{\min }\right)^{-1} .
$$

For the simplified case $s_{k}$ is just the single interval $\left[x_{k 1}, x_{k 2}\right]$ where

$$
\begin{aligned}
& f\left(x_{k 1}\right)=f_{k} \pm \frac{\epsilon_{n}}{2}, \\
& f\left(x_{k 2}\right)=f_{k} \mp \frac{\epsilon_{n}}{2}
\end{aligned}
$$

so

$$
\mu\left(s_{k}\right)=\left|\left(x_{k 2}-x_{k 1}\right)\right| .
$$

Now all that remains is to express $\mu$ in terms of $\epsilon$. This may be achieved by the use of a (functional) Taylor series: 


$$
\begin{aligned}
\epsilon_{n}= & \left|\left(f\left(x_{k 2}\right)-f\left(x_{k 1}\right)\right)\right| \\
= & \mid\left[\left[f_{k}+f^{\prime}\left[f^{-1}\left(u_{n, k}\right)\right] \frac{\mu\left(s_{k}\right)}{2}\right]\right. \\
& \left.\quad-\left[f_{k}-f^{\prime}\left[f^{-1}\left(u_{n, k}\right)\right] \frac{\mu\left(s_{k}\right)}{2}\right)\right] \mid \\
= & \left|f^{\prime}\left(f^{-1}\left(u_{n, k}\right)\right)\right| \mu\left(s_{k}\right) .
\end{aligned}
$$

For a general functional an inf or sup must be taken over the set $f^{-1}\left(u_{n, k}\right)$. Solving for $\mu\left(s_{k}\right)$ and substituting into the expression (A6) for $\rho$ while letting $\epsilon_{n} \rightarrow d u$ and $f\left(x_{0}\right)=u$ gives

$$
\begin{aligned}
\rho(u) d u & =A \lim _{n \rightarrow \infty} \frac{\epsilon_{n}}{\left|f^{\prime}\left(f^{-1}\left(u_{n, k}\right)\right)\right|} \\
& =\frac{A}{\left|f^{\prime}\left(x_{0}\right)\right|} d u .
\end{aligned}
$$

Further insight into the case of a general functional may be gained by first considering a function, $f\left(x_{1}, x_{2}, \ldots, x_{m}\right)$ of $m$ variables. In a small region $\mathbf{U}$ about $\mathbf{x} \in R^{m}$ the value of $f(\mathbf{x})$ is given approximately by

$$
f(\mathbf{x}+\epsilon) \approx f(\mathbf{x})+\nabla f \cdot \epsilon
$$

so there are $m-1$ directions in which $f(\mathbf{x})$ does not change. For $|\epsilon| \ll 1$ the measure may be factored:

$$
\mu\left[f^{-1}(\mathbf{U})\right]=V_{m-1}|\epsilon| \text {. }
$$

Therefore a lower bound for $\rho(u)$ is given by

$$
\rho(u) d u \geq \frac{A V_{m-1}}{|\nabla f|_{\max }} d u,
$$

where $V_{m-1}$ is the $(m-1)$-dimensional volume in $R^{m}$ orthogonal to $\nabla f$ and $|\nabla f|_{\max }$ is the maximum of $\nabla f$ over the small volume. Given this bound it remains true that $\nabla f=0$ is a necessary condition for $\rho(u)$ to be divergent over a compact region of $R^{m}$, since only then does the denominator vanish in the limit as $|\epsilon| \rightarrow 0$. But $\nabla f=0$ is no longer sufficient unless $f(\mathbf{x})$ increases with distance $r$ away from the extremum faster than $r^{m-1}$. If $f(\mathbf{x})$ grows more slowly than $r^{m-1}$ then the volume term $V_{m-1}$ decreases toward the extremum faster than the inverse gradient diverges. However, with the exponential dependence of a Wiener integral, $f(\mathbf{x})$ grows fast enough in any number of dimensions.

For a functional $S[x], \rho(u)$ is inversely proportional to the measure of the functional derivative

$$
\mu\left(\frac{\delta S}{\delta x(\tau)}\right) \text {. }
$$

Again there will be only one direction in function space in which $S$ is changing so the reciprocal of Eq. (A17) will separate from the remainder of a function-space volume element. Whether or not the measure decreases fast enough toward the extremum for the volume contribution to $\rho$ to overwhelm the diverging inverse derivative depends on the measure used.
*Present address: Department of Physics, Utah State University, Logan, Utah 84322.

${ }^{1}$ H. Weyl, Sitz. Ber. Preuss. Akad. Wiss. 26, 465 (1918). Reprinted in The Principles of Relativity (Dover, New York, 1922), Chap. IX; H. Weyl, Space Time Matter (Dover New York, 1952), Chaps. II and IV; H. Weyl, Ann. Phys. 54, 117 (1918); Phys. Z. 21, 649 (1921); Ann. Phys. 65, 541 (1921); Nature 106, 781 (1921).

${ }^{2}$ A. Einstein, Sitz. Ber. Preuss. Akad. Wiss. 26, 478 (1918), including Weyl's reply.

${ }^{3}$ F. London, Z. Phys. 42, 375 (1927).

${ }^{4}$ J. S. Bell, Physics 1, 195 (1965); J. F. Clauser and A. Shimony, Rep. Prog. Phys. 41, 1881 (1978).

5J. Audretsch, Phys. Rev. D 27, 2872 (1983).

6J. Audretsch, F. Gähler, and N. Straumann, Commun. Math. Phys. 95, 41 (1984).

${ }^{7}$ D. Hochberg and J. T. Wheeler, Bartol Research Institute Report No. BA-89-24, 1989 (unpublished).

8 J. A. Schouten and J. Haantjes, Koninkl. Ned. Akad. Wetenschap. Proc. 43, 1288 (1940).

${ }^{9}$ S. Ferrara, Nucl. Phys. B77, 73 (1974).
${ }^{10}$ N. Wiener, Proc. Nat. Acad. Sci. USA 7, 253 (1921); 7, 294 (1921); J. Math. Phys. MIT 2, 131 (1923); Proc. London Math. Soc. 22, 454 (1924); N. Wiener, Acta Math. 55, 117 (1930), Sec. 13; L. S. Schulman, Techniques and Applications of Path Integration (Wiley, New York, 1981).

${ }^{11}$ M. Kac, Probability and Related Topics in Physical Sciences (Interscience, New York, 1959), Chap. IV.

${ }^{12}$ F. W. Wiegel and J. Ross, Phys. Lett. 84A, 465 (1981); K. L. C. Hunt and J. Ross, J. Chem. Phys. 75, 2 (1981); F. Langouche, D. Roekaerts, and E. Tirapegui, Physica 97A, 195 (1979).

${ }^{13}$ E. Nelson, Quantum Fluctuations (Princeton University, Princeton, NJ, 1987).

${ }^{14}$ P. A. M. Dirac, Proc. R. Soc. London A209, 291 (1951); A212, 330 (1952).

${ }^{15}$ R. M. Wald, General Relativity (University of Chicago, Chicago, 1984), Chap. 13.

16 J. Ehlers, A. E. Pirani, and A. Schild, in General Relativity, edited by L. O'Raifeartaigh (Oxford University, Oxford, 1972). 